\title{
¿QUÉ EXPERIENCIAS, ACTITUDES Y COMPORTAMIENTOS TIENEN LOS ADOLESCENTES ESPAÑOLES ANTE LA CONTRACEPCIÓN?
}

\author{
Oltra Rodríguez, Enrique*; Riestra Rodríguez, Rosario** \\ Chamizo Vega, Carmen*; González Pisano, Ana Covadonga*** \\ González Pisano, Marta*; Mirón Ortega, Ma Dolores* \\ Mendiolagoitia Cortina, Luis* \\ * Enfermeras/os Atención Primaria, Asturias \\ ** Enfermera Hospital Universitario Central de Asturias. Área Materno Infantil \\ *** Enfermera Atención Primaria, Villablino (León)
}

\section{WHAT EXPERIENCES, ATTITUDES AND BEHAVIOURS DO SPANISH TEENAGERS HAVE TOWARDS BIRTH-CONTROL?}

\begin{abstract}
It is known that teenagers have a low rate of birth-control methods use in their sexual intercourses which places them in a situation of risk in regard to unwanted pregnancy and sexually transmitted diseases. Although the information they have usually comes from non-formal sources -other peers- this does not seem to explain the rejection -when it comes to intimacy-and the non use of these methods. Facing all this because of the importance that a deep knowledge of this question has for the planning of effective corrective measures, in this article we propose a bibliographic research that should find the hidden factors relating to the real attitudes of teenagers towards birth-control, as well as the cultural environment concerning teenage sexuality, since it may be explicative of the phenomenon described.
\end{abstract}

KEY WORDS: Birth-control, teenagers, attitudes, culture, anthropology

\section{RESUMEN}

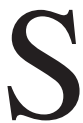
egún varios autores los adolescentes tienen un bajo índice de uso de anticonceptivos en sus relaciones coitales, lo que les coloca en situación de riesgo tanto cara a los embarazos no deseados como a las enfermedades de transmisión sexual. Aunque su información sobre anticonceptivos suele provenir de fuentes no formales, princi- palmente de otros pares, no parece que esto explique la no aceptación -en la intimidad- y el no uso de los mismos. Ante ello y por la importancia que el conocimiento profundo de la cuestión tiene para el planteamiento de medidas correctoras eficaces, en el presente trabajo se plantea una revisión bibliográfica sobre las actitudes de los adolescentes españoles ante la anticoncepción, así como el entorno cultural que envuelve la sexualidad adolescente y que puede ser explicativo del fenómeno descrito.

PALABRAS CLAVE: Anticoncepción, adolescentes, actitudes, cultura, antropología.

\section{MATERIAL Y MÉTODO}

Entre los meses de Noviembre de 2001 y enero de 2002 se ha realizado una revisión bibliográfica del tema señalado en los archivos de diversas instituciones dedicados a la atención específica a jóvenes, en páginas Web y en las bases de datos que a continuación se detallan: IME, Cuidatge, Cuiden, Medline, BIREME (Adolec), CINAHL, ISBN, Centro de documentación de Madrid, SARES (Servicio aragonés de educación para la salud), Escuela Andaluza de Salud Pública, INDEX y CSIC (ISOC - Ciencias sociales y humanidades). Debido al objetivo del trabajo se desecharon los artículos que no se referían a la realidad española, con una excepción por interés específico y que se reseña en el texto.

Los descriptores utilizados fueron: Adolescentes, contracepción (y/o anticoncepción), antropología (y/o cultura) y actitudes. 
Se obtuvieron 47 artículos publicados entre los años 1994 y 2001, de los cuales 33 se han referenciado explícitamente en el texto.

\section{INTRODUCCIÓN}

\section{Adolescencia}

La adolescencia es un proceso, no un estado, una etapa difícil en el camino hacia la madurez, un continuum transicional, en el que se suceden importantes descubrimientos y transformaciones físicas, emocionales y comportamentales de gran impacto en el propio adolescente, en su familia y en todo su entorno. Uno de cada seis españoles es adolescente, es decir, en España hay unos siete millones de adolescentes.

Definir la adolescencia no es una tarea sencilla y aunque ha sido emprendida por múltiples autores y organismos, en la actualidad aún no está concluida y probablemente nunca llegue a estarlo. La adolescencia es una cuestión poliédrica y por tanto el enfoque del que se parta también lo debe ser. Dentro de las diversas definiciones de adolescencia, se pueden destacar a modo de ejemplos las siguientes:

La Real Academia Española de la Lengua, define la adolescencia como la edad que sucede a la niñez y que transcurre desde la pubertad hasta el completo desarrollo del organismo.

El catedrático de sexología Felix López en su libro "Para comprender la sexualidad" dice que la adolescencia es un periodo de la vida en el que se



producen una serie de cambios biofisiológicos, psicológicos, intelectuales y sociales que sitúan al individuo ante una nueva forma de vivenciarse a sí mismo y a todo lo que le rodea".

Tampoco faltan los escritores y poetas que desde su perspectiva han tratado de definir esta etapa, entre ellos Antonio Gala, quien dice "La adolescencia es una edad que no inspira ternura, porque es incomprensible y tiene sin embargo mucho que comprender; porque es incomprensiva y ha de comprender todo, incluida a ella misma. Es una edad tácitamente maldita que no se echa de menos, y suele sonreírse al hablar de ella. La edad del pavo la llamamos".

También la Organización Mundial de la Salud (OMS) a través de su Comité de Expertos en Necesidades de Salud de la Adolescencia, considera adolescencia al grupo de edad de 10 a 19 años, aunque hoy, hasta estos límites están siendo cuestionados y no cabe duda de que existe una amplia variación según analicemos el fenómeno en diversos entornos históricos, étnicos, culturales, geográficos o sociales, además de todos los factores individuales.

Incluso en nuestro entorno sociocultural los límites de este periodo no son estáticos y por ejemplo la actual crisis económica, el desempleo juvenil y otras características de la llamada sociedad del bienestar prolongan mucho la dependencia económica de los jóvenes respecto de sus familias de origen y esto retrasa la ruptura funcional de los adolescentes. Paradójicamente la maduración biológica e intelectual y el distanciamiento afectivo respecto a la familia es cada vez más precoz. Así pues se ven obligados a vivir prolongadamente un estado de adolescencia forzosa, en ocasiones social y personalmente patológico por su excesiva dilatación en el tiempo.

$\mathrm{El}$ adolescente trata de desarrollar la confianza en si mismo, los propios límites y valores, la autodisciplina, sus señas de identidad... en resumen trata de alcanzar la individualidad y la madurez personal. Para todo ello y para "cortar con el cordón umbilical" que le viene uniendo a su familia durante la infancia, el adolescente se sirve, entre otros recursos, del conflicto; que en cantidad moderada y dentro de unos límites aceptables que no pongan en peligro elementos fundamentales de 
su vida (tanto físicos como emocionales o sociales), se puede interpretar como una forma correcta de realizar el proceso.

Dentro del contexto del desarrollo psicosocial del adolescente, la maduración de la sexualidad juega un rol decisivo. La vivencia de la sexualidad en esta etapa dependerá de factores psicológicos, de desarrollo físico individual y de cómo el adolescente interiorice el marco sociocultural en el que se encuentra.

El inicio de esta etapa (la pubertad) con todos sus cambios fisiológico-hormonales implica el comienzo de una intensa vivencia de la sexualidad con múltiples aspectos y sentimientos relacionados, que según su desarrollo, manifestación y adaptación, influyen en todo el ámbito personal y social del adolescente, cuya repercusión se prolonga incluso hasta su vida adulta.

La adolescencia es un periodo decisivo de la evolución sexual, donde el hombre y la mujer a medida que transcurren esos años tienden a cuestionar y alejarse de las normas familiares, para establecer una actitud personal en base a un razonamiento propio.

La actitud de rebeldía independizante, de desafío hacia sí mismos, hacia los demás y la exploración de los límites hace que los adolescentes presenten una atracción por el riesgo difícilmente evitable, siendo todo ello utilizado como estrategia de autoafirmación. Este aspecto está especialmente marcado en lo referente a la sexualidad. Dicha tendencia, junto con su connatural inexperiencia que les hace desconocer muchas variables que influyen en dicho riesgo, provoca que los más jóvenes constituyan un grupo especialmente expuesto, poniendo en peligro en no pocas ocasiones el propio desarrollo y hasta la integridad física, esta realidad se observa, incluso aumentada, en los más desfavorecidos socialmente.

En cuanto a las vivencias de su sexualidad y respecto a la exposición de riesgos también habrá que considerar: la edad de inicio de las relaciones sexuales, que estas son más esporádicas y generalmente no con una pareja estable, y que tienen más dificultades para acceder a métodos anticonceptivos y de protección. A éstas circunstancias, se suman otras de índole social y cultural como pueden ser la oposición de sus padres y de la sociedad en general que no aceptan su activa sexualidad y le dan categoría de tabú, la asunción por parte de los jóvenes de los roles asimétricos asignados a cada sexo, su dificultad para manejarse con habilidades psicosociales del tipo de la asertividad, la negociación, la búsqueda de recursos, etc.

El hecho de que los jóvenes, por reflejo de su entorno adulto, no se reconozcan como sexualmente activos hace que no busquen ni la atención necesaria ni la educación adecuada.

\section{Actitudes}

Según la Real Academia Española de la Lengua "actitud" es la disposición de ánimo manifestada exteriormente, postura que se adopta frente a una idea o acción.

Las actitudes del adolescente frente a la sexualidad al estar determinadas en su mayoría por criterios sociales y antropológicos, tienen gran variabilidad de tipo local (según el país o la región), temporal (según la época o momento histórico) y también personal que dependerá de cómo la persona interiorice los anteriores y estará condicionada a la edad, al nivel económico y cultural, y a los conceptos particulares de orden filosófico, ético, moral, religioso...

En cuanto a criterios morales que condicionan las actitudes sexuales de los adolescentes se observa variabilidad según la etapa que viven: adolescencia temprana (10 a 13 años), etapa media (14 a 17 años) y tardía (superior a 18 años); en base a ello el Dr. Kohlberg describió su Desarrollo Moral dividido en 3 períodos:

- Pre-Convencional: Característico del pre-adolescente donde las interpretaciones son en base a lo inculcado por su familia (concepto de bueno-malo reforzado con premio-castigo).

- Convencional: Se observa en el adolescente temprano y medio, todavía mantiene un respeto a las reglas familiares, como expresión de acatar el orden social.

- Post-Convencional: Se presenta al final de la adolescencia, la persona tiene ya conceptos propios y toma decisiones independientes.

\section{Anticoncepción y adolescentes}

La planificación familiar es el derecho de las personas a regular su fertilidad, tiene tres fundamentos principales que son: los derechos humanos, 
los aspectos relacionados con la salud y la demografía. Experimenta un avance crucial con la contracepción hormonal, denominada "revolución contraceptiva", que en España no se legaliza hasta 1978. Para la OMS, la planificación familiar es una forma de pensar y vivir, adoptada voluntariamente por individuos y parejas, basada en conocimiento y actitudes de cara a promover la salud y el bienestar de la familia y de la comunidad.

Métodos anticonceptivos: son aquellos instrumentos, fármacos o procedimientos que evitan que las relaciones sexuales vayan ligadas a la reproducción, impidiendo que un óvulo sea fecundado por un espermatozoide. No existe ningún método perfecto que vaya bien a todas las personas y en todas edades y situaciones.

En relación con la adolescencia, la FIGO (Federación Internacional de Ginecólogos y Obstetras) clasifica los métodos anticonceptivos para este grupo de edad en:

Preferentes: preservativo y contracepción hormonal oral.

Aceptables: diafragma, esponja, espermicidas e inyectables.

Menos aceptables: DIU, abstinencia periódica y contracepción quirúrgica.

De emergencia: contracepción postcoital.

Bajo el punto de vista de la joven usuaria, los métodos anticonceptivos han de: 1- ser seguros para su privacidad (además del embarazo, han de evitar que sus relaciones sexuales sean descubiertas por quienes se oponen a ellas). 2- ser eficaces y aceptables (que encajen en el clima romántico y sean compatibles con el rol que la joven desarrolla en la relación; no pueden amenazar la identidad ni la magia del encuentro sexual). 3- se han de presentar como relevantes para la situación de noviazgo y no solo para evitar la procreación o la infección.

\section{EL ESTADO DE LA CUESTIÓN: (DATOS SOCIOLÓGICOS Y EPIDEMIOLÓGICOS)}

¿Qué conocen sobre sexualidad y anticoncepción los adolescentes?

Los adolescentes creen en un alto porcentaje $(47 \%)$ tener suficiente información sobre los aspectos relacionados con la sexualidad. Otros estudios encuentran que hasta el $73 \%$ de los jóvenes se considera bien informado en temas relacio- nados con la sexualidad, sin embargo se objetiva una diferencia entre lo que creen saber y lo que realmente saben, lo que provoca una baja percepción de riesgo y una errónea actitud preventiva.

A pesar del punto en el que podamos creer que estamos, los adolescentes ni tan siquiera reciben información (no se habla ya de formación) ni de la familia ni de la escuela, las chicas reciben información fundamentalmente de las amigas/os, mientras que los chicos la obtienen de los amigos y también de libros, revistas y medios de comunicación. El contexto social influye determinantemente en el nivel de conocimiento.

El origen de la información sexual recibida es: En los chicos, la televisión 47,5\%, los amigos y amigas $44,2 \%$, las revistas $25,2 \%$ y madre $11,9 \%$. En las chicas, las amigas y amigos $43,8 \%$, las revistas $31,4 \%$, la televisión $26,5 \%$ y la madre $19,3 \%$. En otro estudio realizado por la junta de Andalucía las fuentes informativas eran por orden de importancia: amigos $(27,8 \%)$, el cine y la televisión $(22,9 \%)$, las madres $(17,7 \%)$, revistas o libros $(16,4 \%)$ y el profesorado $(15,2 \%)$. El estudio CIS 1992 concluye que la información sexual que reciben los jóvenes es escasa, aunque como es lógico pensar, también se encontró que con la edad disminuye la desinformación.

Sobre qué información demandan los adolescentes al ser preguntados, hay diversidad de opciones según las publicaciones consultadas, pudiendo deberse, entre otras causas, al método utilizado (quién hace la pregunta, en qué contexto, tras qué intervención...), además las respuestas son en general muy tópicas y genéricas. En un estudio realizado a 276 adolescentes (entre 14 y 18 años) en el I.B. "La Baseta de Beniguasil, se observa un claro predominio del interés por los temas relacionados con la sexualidad $(91,55 \%)$ frente a otros temas como drogas, alcohol, tabaco, cáncer e higiene personal. De entre los temas de sexualidad interesaba en primer lugar la sexualidad humana $(29,7 \%)$, seguido de las I.T.S $(31,15 \%)$ y el embarazo $(30,7 \%)$. En un cuestionario realizado a los alumnos de $4^{\circ}$ ESO en Sevilla en 1994 los principales temas de interés eran: los embarazos no deseados, los anticonceptivos, anatomía, masturbación, la primera vez y ciclo de respuesta sexual. 
Es interesante destacar que el interés que suscitan los temas de sexualidad se desplazan según la edad: los más jóvenes prefieren contenidos de sexualidad humana (un reflejo del comienzo de su actividad sexual) frente a los grupos de más edad que optan por temas de prevención del embarazo e infecciones de transmisión sexual (I.T.S.).

Hasta el momento sólo se está abordando la cuestión del conocimiento, de la información, conscientes de que conocer los métodos anticonceptivos no evita los embarazos entre adolescentes como asegura el experto en educación para la salud sexual José Cáceres, que cree más en la necesidad de que los jóvenes sepan comunicarse y negociar estos temas sexuales, de cara a eludir sus consecuencias negativas.

\section{¿Qué usos sexuales tienen los adolescentes?}

La coitarca o edad de la primera relación sexual con penetración varía mucho según los estudios consultados y se encuentra como media estadística entre los 16 años y los 17,9. Aunque es un dato significativo que sobre un $25 \%$ de los adolescentes ya han practicado coito a los 15 años, elevándose a los 18 años entre un $56,9 \%$ y un $76,96 \%$. Siempre se aprecia un ligero retraso en la edad de las chicas respecto a los chicos.

En cuanto a la razón más importante para decidir mantener la primera relación sexual, las chicas refieren "porque estaba enamorada" en un 76,5\%, mientras que los chicos refieren esta respuesta en un $47,3 \%$. En los chicos también es significativo que un $20,9 \%$ responden "me gustaba aquella persona aunque no estaba enamorado de ella" y otro $20,9 \%$ indican como razón "tenía ganas de tener una relación sexual con penetración”. Es decir, las adolescentes en sus relaciones de pareja muestran una clara preferencia por los aspectos afectivos mientras que ellos valoran más la genitalidad y el sexo en sí mismo.

Carmen de la Cuesta, en una de las pocas investigaciones cualitativas que hemos encontrado en la bibliografía, aunque realizado en Colombia, refiere: Parece que la joven toma la decisión de iniciar relaciones sexuales de manera privada y aislada del mundo social. Para la joven, el experto es "el novio en serio" que hace de maestro en la relación. Aunque este estudio fue realizado entre jóvenes de
Colombia, es muy posible, aunque no lo podemos confirmar, que lo encontrado sea trasladable a la realidad española.

Además los adolescentes, al igual que los adultos, pueden iniciar o mantener sus relaciones sexuales por motivaciones ajenas a sus necesidades físicas o emocionales. Algunas de estas motivaciones pueden ser:

- Desafiar la norma social, por la existencia de la doble moral que por un lado favorece la liberación de las costumbres y por otro lado lo critica.

- Ser aceptado por el grupo y que no le marginen, la presión negativa de las amistades puede fomentar conductas tendentes al inicio de la actividad sexual completa.

- Enfrentar los sentimientos de soledad, baja autoestima o el rechazo de otra pareja.

- Huir de la familia (sobre todo los que tienen un ambiente familiar conflictivo), suelen buscar en la relación de pareja la protección y el afecto que no encuentran en otro ámbito. Para reforzar sus lazos, acceden a mantener relaciones sexuales, aunque no sea una necesidad sentida.

- Confirmar y reafirmar su femineidad o masculinidad, el adolescente cree que practicar el sexo lo hará más hombre o más mujer.

Respecto al cómo, hay que destacar una gran improvisación. El grado de anticipación de la primera relación es escaso (el 70\% de los chicos y el $60 \%$ de las chicas no lo anticiparon), pues dicen que sus relaciones "ocurren espontáneamente", este hecho hace más difícil el uso de anticonceptivos. En éste mismo estudio, los adolescentes comunican que el hecho de no haber mantenido relaciones coitales aún, se debe a "no haber encontrado a la persona adecuada" y a "no haber tenido ocasión". En un grado mucho menor (6\%) al miedo al embarazo y a las enfermedades de transmisión sexual.

$\mathrm{Y}$ respecto al dónde mantienen sus relaciones sexuales los jóvenes, según el estudio del sexólogo J.L. García son: Casa ajena $(64,3 \%)$, coche $(13,3 \%)$, pensión u hotel $(0,9 \%)$, parque o portal $(0,8 \%)$ y otros $(20,7 \%)$.

\section{¿Qué uso de anticonceptivos hacen los adoles- centes?}

Entre la primera caricia (inicio de relaciones 
sexuales) y el primer coito transcurren entre 12 meses y 24 meses y entre el primer coito y el uso de forma más o menos regular de algún método anticonceptivo pasan también entre 12 y 24 meses. Entre los adolescentes que consultaron en un centro de planificación familiar, se encontró que tardaron en acudir más de un año desde su primer coito.

Según algunos estudios, entre un $22 \%$ y un porcentaje superior al $50 \%$ de los adolescentes no usaron ningún método anticonceptivo en su primera relación sexual.

Todas estas secuencias son de gran trascendencia a la hora de programar intervenciones preventivas de riesgos, pues aproximadamente la mitad de los embarazos no deseados en adolescentes ocurren en los primeros meses de relaciones sexuales.

Entre un $19 \%$ y un $45,41 \%$ no los usan habitualmente o utilizan uno de los considerados no seguros.

En un grupo de adolescentes embarazadas solo habían utilizado algún método anticonceptivo el $11,9 \%$, a pesar de lo cual éstas también quedaron embarazadas por utilizar alguno de baja fiabilidad (coitus interruptus o métodos del ritmo) o bien por no utilizarlo adecuadamente (preservativo), observándose sobre todo una falta de constancia en el uso del mismo. También en un grupo de mujeres menores de 21 años que realizaron una IVE, el $45,41 \%$ no estaban usando ningún método anticonceptivo o usaban métodos considerados muy poco seguros (Ogino, coitus interruptus...), el 5,34\% usaban AHO y el $49,98 \%$ utilizaba el preservativo (el 13,37\% no lo usaba correctamente y el 35,55\% sufrió una rotura).

Sin que se establezcan diferencias en función del sexo, los jóvenes afirman que utilizarían el preservativo en un $95 \%$ de los casos. Pero estas "buenas intenciones" no parecen guardar relación con la mayor parte de los datos encontrados.

Entre la percepción que los jóvenes tienen de estar usando un método eficaz y el que este realmente lo sea, existe una notable diferencia, de los jóvenes que creían utilizar un método seguro $(76 \%)$, sólo la mitad lo hacía realmente, pues un $38 \%$ practicaba el coitus interruptus y otro $12 \%$ tenían errores en la técnica o no eran constantes en el uso.

En lo referente el uso de Centros de
Planificación familiar, el perfil de adolescente que lo utiliza es el de una chica de 17,6 años de edad, soltera, estudiante, con menarquia a los 12,5 años, sin infecciones ni gestaciones previas que acude al CPF sobre el primer año de mantener relaciones sexuales. No descubrimos nada nuevo si decimos que en nuestra sociedad la responsabilidad contraceptiva se ha delegado siempre en la mujer; y con ésta el riesgo de embarazo y las consecuencias transcendentales que se derivan del mismo.

Respecto a la contracepción postcoital, las mujeres jóvenes son las que la solicitan mayoritariamente, en concreto el $39 \%$ de las demandantes de intercepción postcoital son adolescentes menores de 20 años y de estas el $29 \%$ reinciden. Los motivos de solicitud de la píldora postcoital declarados por las usuarias son: $62,93 \%$ rotura de preservativo, $17,76 \%$ retención de preservativo en vagina y $19,30 \%$ no uso de anticonceptivo, esto contradice la realidad reflejada en múltiples estudios y está más en relación con lo esperado como correcto que con lo real.

Los adolescentes tienen poco acceso a la atención contraceptiva (recursos) y a la información/ orientación/tratamiento psico-sexual. Esto se debe en los servicios privados a la baja capacidad adquisitiva de los adolescentes y en el caso de los servicios públicos a su escasez y saturación, así como a su difícil accesibilidad para los jóvenes por su estructura (burocracia, horarios...) orientada hacia los adultos. En los últimos años, los centros de salud están adoptando medidas que aumentan la accesibilidad de los adolescentes, organizando servicios, como las llamadas "consultas joven". Estas experiencias son todavía escasas y cubren las necesidades de muy pocos adolescentes.

\section{ACTITUDES DE LOS ADOLESCENTES ESPAÑOLES ANTE LOS ANTICONCEPTI- VOS:}

Las actitudes de los jóvenes hacia los anticonceptivos están íntimamente ligadas a las actitudes y creencias con que viven su sexualidad en general y esta se configura sobre tres elementos: el modelo socio-cultural, la historia afectivo-familiar y los procesos de identidad específicos de esta etapa de la vida.

Los jóvenes desarrollan guiones de comporta- 
miento desde lo escuchado a otros, especialmente su familia y su grupo de iguales, de absorber la cultura de su medio inmediato, pero los medios de comunicación y las nuevas tecnologías también cobran relevancia, como referentes, en la construcción de guiones de vida. La influencia de cada referente en el constructo de la sexualidad de los jóvenes es algo que indudablemente merecería ser explorado.

\section{Actitudes desde la individualidad del adolescente}

Las relaciones sexuales entre adolescentes no suelen estar previstas o no se ajustan a las expectativas creadas. A esta falta de premeditación y programación consustancial a su edad, hay que sumar que en ocasiones las relaciones sexuales se producen bajo los efectos del alcohol u otras sustancias tóxicas, suponiendo la alteración de la conciencia que producen, una dificultad sobreañadida para el uso de medios anticonceptivos. También debido a su edad, también pueden presentar dificultades en su capacidad asertiva (dificultad para decir "no") y de negociación.

Las razones para la no utilización de métodos anticonceptivos por parte de lo adolescentes es muy variable, entre ellas cabe destacar que el 50\% de ellas no lo hicieron porque no esperaban tener relaciones sexuales, e incluso el $21,8 \%$ no lo usaban por creer peligroso o desacertado el propio método. Entre las causas para no acudir a un centro sanitario para solicitar información o métodos, el $40 \%$ argumentaron no conocer la existencia de los mismos y el $35 \%$ manifestaron temor a verse descubiertos por sus progenitores.

Aunque no hemos encontrado estudios al respecto con adolescentes españoles, en el realizado por Carmen de la Cuesta Benjumea en Colombia, encontró que el embarazo (entendamos este como la situación de no anticoncepción) se produce en un contexto de interacción que podemos denominar de "noviazgo en serio" en el que las ideas de amor romántico y las reglas de género (aceptación e interiorización de un convencimiento sobre el rol femenino o masculino, que corresponde asumir en las relaciones sociales) guían su comportamiento. Se puede decir que el amor romántico es la cultura de las jóvenes en su relación con un novio en serio. Las relaciones sexuales se van a utilizar para demostrar confianza y por otra parte para dar esta- bilidad a la relación.

Existe una influencia de la interiorización de los roles masculino y femenino, y existe una diatriba entre los chicos que tratan de convencer a las chicas para "que lo hagan" y éstas que por una parte tienen curiosidad y ganas y por otra tratan de "resistirse" por miedo, inseguridad ("quizás no sepa hacerlo y quede en ridículo", "no te puedes fiar de ellos"), vergüenza (falta de identificación con la propia imagen corporal), prejuicios sociales ("luego te consideran una chica fácil") y morales e incluso asco por desconocimiento de la genitalidad y del proceso costal.

En algunas chicas también se observa una cierta identificación entre sexo y maternidad idílicamente concebida, pudiendo encontrarse en ello la causa de algunos embarazos que en principio pudieran parecer no deseados y que aunque "inoportunos", son buscados.

Los adolescentes, dentro de su concepción de invulnerabilidad e incluso de inmortalidad, están convencidos de estar protegidos por una especie de magia, sólo posible en su mundo particular de relaciones. No debemos esperar que el miedo al "castigo" del embarazo no deseado o incluso a las I.T.S., vayan a intimidar a los jóvenes que deciden aventurarse en el encuentro genital. Infravaloran su potencialidad reproductiva e incluso tienen déficits informativos. Un estudio clásico de Shah, F. y col. pone de manifiesto, que el $70 \%$ de las chicas entre 15 y 19 años no creían poder quedarse embarazadas.

También aparece un alto grado de coherencia en las respuestas cuando se correlacionan las actitudes, medidas con diferentes escalas, hacia la sexualidad y sus comportamientos sexuales. En este sentido un $68,9 \%$ de los chicos y un $37,4 \%$ de las chicas muestran una actitud negativa o muy negativa hacia la sexualidad, y entre este grupo de jóvenes es más frecuente encontrar a quienes reconocen no haber utilizado ningún medio anticonceptivo en la primera relación, no utilizarlos en las relaciones actuales, no consultar a profesionales de la salud cuestiones relacionadas con la sexualidad o la anticoncepción, no percibir el riesgo de contraer I.T.S. y tener un bajo control emocional.

\section{Actitudes ancladas en la realidad familiar}

Se encuentran muy pocas referencias a la influencia de las familias sobre las cuestiones a 


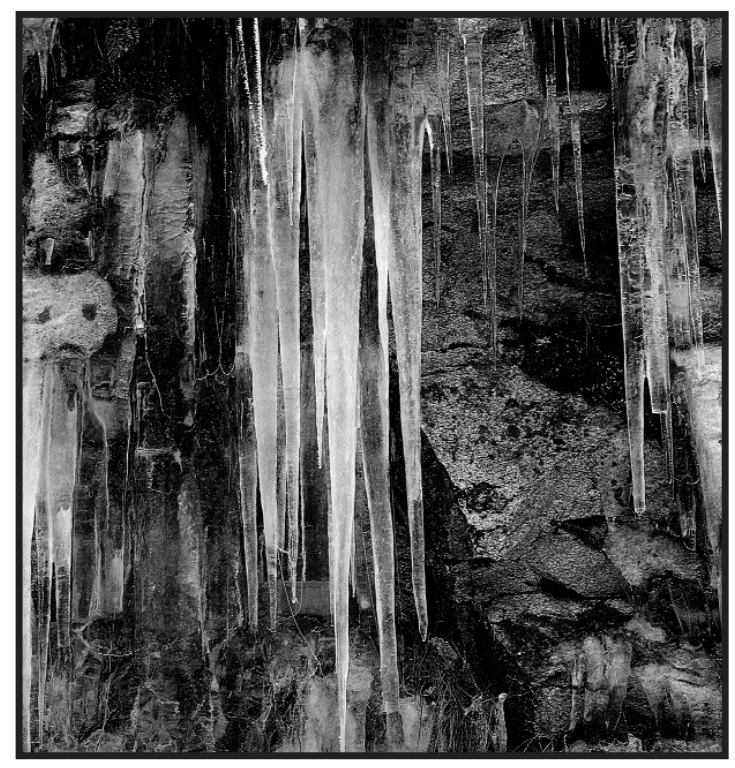

estudio, parece que los actuales hijos e hijas adolescentes tienen problemas similares a los que sus madres y padres tuvieron en su momento, las emociones y sentimientos no cambian tanto aunque hayan pasado los años y las generaciones. Los noviazgos suelen tener la aprobación de los padres que legitiman la relación sentimental, no sucediendo lo mismo con las relaciones sexuales en general. La actitud familiar respecto a la sexualidad adolescente evoluciona mucho más lentamente que la que se percibe en las estructuras sociales y que transmiten los medios de comunicación.

En cuanto a la relación existente entre la estructura del grupo familiar y las relaciones sexuales encontramos los siguientes datos:

Hijos de familia donde falta el padre o la madre (solteros o viudos) han tenido relaciones sexuales el $66,7 \%$ de la muestra, con padres separados o divorciados las han tenido el $53,8 \%$ y con padres casados el $41,9 \%$. Esto se observa igualmente ajustando los datos por sexos o por edades. Sin embargo, el nivel de protección anticonceptiva es casi un $15 \%$ mayor en el primer grupo que en el segundo y en el tercero.

Los adolescentes con problemas familiares y/o de integración social tienen relaciones sexuales antes y en muchas ocasiones buscan el embarazo como salida a situaciones personales negativas, ven a su futuro hijo como una posibilidad de huir de su situación y crear una nueva familia a la que entregar y de la que recibir afecto.

\section{Actitudes ancladas en la realidad social}

Los jóvenes reciben de su entorno mensajes contradictorios, por una parte un estímulo cuando no una incitación a una liberación sexual y por otra el planteamiento de gran número de dificultades y obstáculos para el desarrollo y práctica de una sexualidad satisfactoria.

El entorno cultural y educacional de la joven va a influir en su conducta sexual de forma importante, así en un estudio estadísticamente significativo entre 2.151 mujeres menores de 20 años que parieron en el Hospital $1^{\circ}$. de Octubre de Madrid, el subgrupo de mujeres escolarizadas utilizaban anticonceptivos el 96,1\%, mientras que en las que se confesaron analfabetas el porcentaje era del $3,98 \%$. La utilización de anticonceptivos también era superior en las mujeres integradas en el mundo laboral que en aquellas desocupadas o dedicadas a sus labores. El 15\% de las adolescentes embarazadas estudiadas, habían tenido ya otros embarazos anteriores (reincidían) lo cual puede apoyar la idea de que al no verse modificado, o verse modificado negativamente, su estatus socioeconómico el factor de riesgo continúa presente. En otra muestra se ha encontrado que el $81 \%$ de las adolescentes embarazadas no estaban escolarizadas ni tenían trabajo.

Aunque se producen embarazos adolescentes en todo tipo de clase social y nivel cultural, las malas y bajas condiciones socio-económicas y culturales son destacados factores que favorecen el inicio precoz de las relaciones sexuales y de los embarazos adolescentes.

UNICEF en su informe de agosto de 2001 refiere que una situación de pobreza, sumada a no ir bien en los estudios incrementa el riesgo de embarazo adolescente y a su vez un embarazo adolescente incrementa el riesgo de caer en la pobreza y de no finalizar los estudios.

Hay una construcción social que considera al embarazo en la adolescencia como una desviación, pues viola normas relativas al embarazo y a la adolescencia. La adolescencia (en nuestra sociedad) es una etapa a medio camino entre la infancia y la madurez y claramente no se considera el momento para la reproducción. El embarazo adolescente es 
estigmatizado socialmente bien sea por la repercusión económica que tiene para el estado (en aquellos países con un estado del bienestar) o bien por una preocupación sobre la moralidad sexual. El rechazo social se hace sentir en la interacción cotidiana de la joven con los otros, ya que la conducta desviada se crea también cuando las personas la perciben e interpretan de esta manera.

Cabría aquí la reflexión de si el rechazo social al fracaso de los anticonceptivos, embarazo adolescente no deseado, es debido a la inoportunidad del mismo o es consecuencia al rechazo moral de la sexualidad adolescente (prematura, precoz, inmoral, inmadura...) que queda evidenciada en la realidad del embarazo. ¿Soporta un análisis histórico, transcultural y/o étnico la tan mencionada “inmadurez psicológica" o la "prematuridad genital" de los adolescentes?

\section{CONCLUSIONES Y DISCUSIÓN}

A la luz de la bibliografía revisada creemos que aunque sea obvio, es necesario destacar que los adolescentes españoles mantienen, de hecho, relaciones coitales, siendo una característica frecuente el que las realicen sin una adecuada protección contra el embarazo no deseado y las I.T.S., cuestiones que no perciben como un riesgo real y para cuya prevención tienen baja capacitación. La sexualidad adolescente continúa siendo socialmente rechazada en nuestro entorno, lo que hace que en muchas ocasiones se ejercite clandestinamente y ni tan siquiera el joven se la autorreconozca. Dichas relaciones no son anticipadas ni mucho menos programadas.

Aunque el riesgo existe en todo estrato sociocultural, parece establecido un claro incremento del mismo relacionado con el nivel y las condiciones sociales, culturales, económicas y de escolaridad.

Además de los déficits de conocimientos y baja integración de los mismos, y de la dificultad de acceso a los medios necesarios, se atisba, aunque no se confirma por no haber sido estudiado explícitamente en ninguno de los trabajos revisados, que la etiología podría estar también en relación con factores tales como: habilidades personales relacionales, aprendizaje de conductas (heredadas de su familia y entorno) y actitudes hacia la sexualidad y todo aquello que la rodea.
La separación entre ejercicio sexual y procreación puede no estar perfectamente clara en la adolescencia, donde en un intento de indagar en los límites y las posibilidades de su nuevo estatus de relación, pero también reproductivo, puede llevarles de una forma más o menos consciente, a pretender explorar su, también nueva, capacidad reproductiva. Además en la conceptualización idílica y bucólica de la relación amorosa entre adolescentes, estos pueden contemplar la posibilidad de una maternidad que complete el cuadro. Así mismo la adolescente -el adolescente tambiéninmersa en las dificultades de encontrar en la familia el ámbito donde cubrir sus necesidades afectivas, puede derivar estos afectos a su pareja con quien desea asegurar y estrechar vínculos, o incluso con el espejismo de su potencial hijo.

Su falta de análisis objetivo -adulto- y la inexperiencia propia de su corto recorrido vital pueden no permitirles percibir las complicaciones consiguientes a una maternidad en ese momento de la vida.

Para comprender las actitudes que presentan los adolescentes actuales ante la sexualidad y la contracepción, hay que tener muy presente el ámbito en que se forjaron las de la generación de sus progenitores, y en el caso español no se debe perder de vista la gran influencia moral que la religión imperante en la época tuvo en el anterior régimen político y que se infiltró en el subconsciente tanto individual como colectivo.

Por otra parte, en poco tiempo se ha producido un gran salto en las aportaciones que la ciencia ha realizado para poder separar actividad sexual de procreación y los anticonceptivos han visto mejorada su eficacia y técnicamente se adaptan cada vez mejor a la edad adolescente, sin embargo estas adaptaciones técnicas no han venido acompañadas por otro tipo de adaptaciones como pueden ser las logísticas para su implementación en dicho grupo, es decir, no se han adaptado a sus características psicológicas, sociales, de madurez, de accesibilidad a los ámbitos donde los pueden conseguir, incluso de aceptabilidad en el entorno del adolescente. La medicalización de la vida íntima y cotidiana a la que está condicionado el acceso a buena parte de los anticonceptivos (venta en farmacias, revisiones y controles, burocracia sanitaria...), dificulta para los adolescentes el acceso a los mis- 
mos, pues la adolescencia es una edad donde la construcción de la imagen corporal aun no está completa ni integrada, con un sentido del pudor sobredimensionado, gran inseguridad en sí mismos y en sus comportamientos..., lo que junto a la rebeldía y la negación de cualquier autoridad, y en este caso no se debe perder de vista que la sanitaria también es reconocida como tal, hacen que los jóvenes, en un alto porcentaje, renuncien a los posibles medios de protección.

La realidad de los adolescentes respecto al uso de los anticonceptivos ha sido profusamente estudiada, pero solo con métodos epidemiológicos y cuantitativos, y desde la perspectiva sanitarista, esto es necesario para empezar a conocer el fenómeno y sobre todo su magnitud, pero no es suficiente.

La interpretación que las jóvenes hacen de sus condiciones o circunstancias y su construcción social, no ha sido prácticamente explorada. La compleja estructura que compone la realidad de comportamientos tan íntimos, como todo lo referente a la sexualidad, y además en poblaciones como la adolescente, es difícilmente accesible para los "investigadores adultos", sin embargo este conocimiento es fundamental para elaborar sobre él estrategias efectivas de abordaje del fenómeno estudiado.

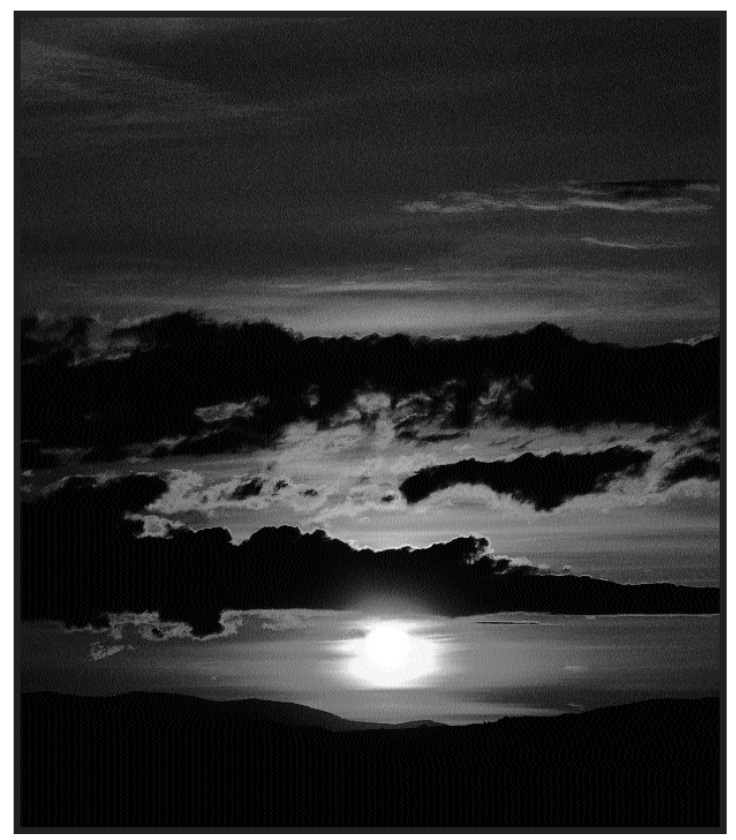

La adecuada utilización de los anticonceptivos por parte de los adolescentes no depende sólo de campañas de divulgación informativa ni tan siquiera de consultas específicas, sino de una compleja serie de factores, en ocasiones no del todo conocidos.

Si como hemos visto las relaciones sexuales suelen estar mayoritariamente ligadas a los afectos y condicionadas por los recursos relacionales individuales, la educación sexual para ser efectiva, también ha de estar unida a la afectividad, fomentando que los jóvenes analicen influencias y actitudes, y que entrenen habilidades.

Los profesionales de la salud, además de otros, debemos asumir el compromiso que nos corresponde en nuestro papel como educadores y facilitadores de dichos procesos. La eficacia de nuestras intervenciones va a estar mediatizada por el grado de comprensión que tengamos del proceso y de las circunstancias condicionantes que lo rodean y para ello es determinante el profundizar con una "mirada antropológica".

Nuestro agradecimiento a Cristina González Aller y a Guillermo Oltra Rodríguez por su apoyo en aspectos de estilo y traducción.

\section{BIBLIOGRAFÍA:}

- Educación para la salud: sexualidad en la adolescencia. Experiencia en el I.B. "La Baseta" de Beniguasil. Revista Enfermería Integral. Junio-Agosto 1.996; 37 : XXXII-XXXVI

- BARRAGÁN MEdero F. (1999) Programa de educación Afectivo Sexual. Educación secundaria. Edita junta de Andalucía. Consejería de Educación y Ciencia. 5A Instituto Nacional de Estadística.

- BARRERA G. KERDEL V. (1980) El adolescente y sus problemas en la práctica. Monte Ávila Editores. Segunda edición. Caracas.

- CABRERA DEL CASTILLO, MA. MARTÍN DÍAZ, J. LUQUÍN AJURIA, A. ORUETA SÁNCHEZ, R. LÓPEZ DE CASTRO, F. (1996) Conocimientos, actitudes y comportamientos sobre sexualidad en dos colectivos de jóvenes-adolescentes. Centro de Salud, Mayo; 297-302.

- CAMACHO GARRIDO, J. DUARTE MERETO, C. DE TENA SERENO T. (1999) Planificación familiar en adolescentes: Objeto de enfermería. Revista Profesión, boletín informativo del Colegio de Enfermería de Sevilla, Julio.

- CARPINTERO, E. (1996) Comportamiento heterosexual y anticonceptivo en adolescentes. Relación con 
riesgo de embarazo no deseado, ETS y VIH. Diálogos. Universidad de Salamanca. Diciembre, 25-28

- CONFORT, A. (1990) Adolescente, sexualidad, vida y crecimiento. Editorial Blume. Barcelona. España.

- DE LA CUESTA BENJUMEA C. (2000) Significado del embarazo en la adolescencia: la dimensión sentimental. Enfermería Clínica. Vol. 10, nº 5. 200-206.

- DE LA CUESTA BENJUMEA C. (2001) Contexto del embarazo en la adolescencia. Revista Rol; 24(9): 584-590.

- DICCIONARIO DE LA LENGUA ESPAÑOLA. REAL ACADEMIA ESPAÑOLA (1992). Vigésima primera edición.

- EL PAÍS (1994) Conocer los métodos anticonceptivos no evita los embarazos entre adolescentes. Lunes 24 de Octubre de 1994.

- FABREGAS RUESGAS, JJ. ROS I RAHOLA, R. (2001) Estudio del comportamiento de jóvenes estudiantes en Barcelona. Diálogos, Revista de la Federación de Planificación Familiar de España. № 46, Enero-Marzo.

- Federación de planificación Familiar de España. (2001) Estudio sobre mujeres menores de 25 años que abortaron en la clínica Dator en 1999. Revista de la Federación de Planificación Familiar de España. № 46, Enero-Marzo.

- GALA, A. (1989) Los adolescentes. El País Semanal.

- GARCÍA-CERVERA J., et als. (1997) Comportamiento sexual, contraceptivo y reproductivo de las adolescentes. La primera visita en un CPF de 1986 a 1996. Diálogos. Universidad de Salamanca. Septiembre; 25-29.

- GARCÍA FERNÁNDEZ, J. L. (1991) Embarazo no deseado en adolescentes: una propuesta de intervención. Revista Rol de Enfermería, Octubre; 158: 36-42.

- GONZÁlez ANTÓN, G. (2000) La FPFE (Federación de planificación familiar de España) y la salud sexual reproductiva de los adolescentes. Revista Diálogos $\mathrm{n}^{\circ} 43$, Junio.

- GUTIÉRREZ LEÓN MaA., MARTÍN ARMAS $M^{\mathrm{a} J}$. (1999) Sexualidad y Juventud. Embarazo no deseado y Métodos anticonceptivos. Marzo. Plan canario de educación y atención a la sexualidad juvenil. Página: http://www.gobcan.es/psc/syjcal.htm

- JIMÉNEZ, JS. HERNÁNDEZ GARCÍA, JM. SÁNCHEZ, D. MONTELIU, P. ESPANTALEÓN, MG. DE LA FUENTE, P. Contracepción en la adolescencia: dificultades, eficacia y resultados. Acta ginecológica, 1994; LI: 139-144.

- LÓPEZ, F. Y FUERTES, A. (1998) Para comprender la sexualidad. Ed. verbo divino. Navarra

- MARCELINO PÉREZ, SS. SÁNCHEZ DE SAN LORENZO, A. MARCELINO PÉREZ, DA. (1997) Embarazo adolescente: una propuesta de intervención.
Revista Rol de Enfermería, Septiembre; 229.

- MARTÍNEZ CABANES, M. (2001) Preservativos y educación sexual. Revista Diálogos. No 48, sept.

- PORTELLA, E. CASTILLO, A. (1998) Aspectos sociales del embarazo en adolescentes. Gaceta Sanitaria; 2:150-153.

- POVEDANO, N. et al. (2001) Educación sexual para padres y madres. Prevención de embarazos en la adolescencia. Revista Diálogos, $\mathrm{n}^{\circ} 48$, sept.

- ROZAS GARCÍA, M. R. (1996) Contracepción postcoital: una opción de emergencia. Rol de Enfermería. Septiembre.; 229: 74-77.

- ROZAS, MR. COSTA, J. OLLÉ, A. HERNÁNDEZ, I. LÓPEZ, F. GRAUC, M. CONDEC, M. NICOL, E. (2000) Intercepción postcoital. Análisis de 200 casos consecutivos. Enfermería clínica, 3; 10: 106-113.

- RUIZ JIMÉNEZ, MA et al. (1997) Adolescencia y anticoncepción. Revista Rol de Enfermería, Junio; 226: 61-64.

- RUIZ JIMÉNEZ, MA. MARTÍNEZ NIETO, JM. FERNÁNDEZ GARCÍA, JR. PAVÓN LEBRERO, R. CANO FERNÁNDEZ, MC. (1997) Anticoncepción en adolescentes incluidas en un programa de planificación familiar. Atención Primaria, 9; 19: 455-458.

- SÁEZ I. (1992) Sexualidad en la adolescencia. Publicación Dirección Materno Infantil MSAS, UNICEF, OPS / OMS. Litobrit. Octubre, Caracas.

- SÁEZ DÍAZ I. (1994) Adolescencia y sexualidad, actitudes y angustias. Ministerio de Sanidad y Asistencia Social. Fundasalud LARA. Editorial Boscan C.A. Venezuela.

- TENORIO CAMPANARIO, P. (1997) Médico de familia y diplomado en sexualidad. Consulta Joven de Orientación Sexual en Centros Educativos.Página: http://www.arconet.es/med/avg/consultajoven.htm

- UNICEF. Noticias de (2001). Madres adolescentes en países ricos. $\mathrm{N}^{\circ} 176$. Agosto.

- YAGO SIMÓN, T. (2001) Educadores y adolescentes en grupo operativo. Revista Diálogos. No 48, septiembre.

\section{Otra bibliografía de interés consultada pero no reseñada en el texto}

- Autor (1991) Embarazo no deseado en adolescentes: una propuesta de intervención. Revista Rol de Enfermería, Octubre; 158: 36-42.

- AGUTZANE ESCALERA URGIAGA ET ALS. (1993) Planificación familiar. Valoración de un programa. Revista Rol de enfermería. Abril; 176: 25-30.

- BOXÓ CIFUENTES, JR ET AL. (1995) Conducta sexual y contracepción en adolescentes escolarizados de la zona básica de salud de Alora, Málaga. Revista Medifam. Marzo-abril; 5(2):75-79. 
- CAMACHO RÍOS, CRISTINA ET ALS. (1996) Embarazo en la adolescencia. Albacete, 1994-1995. Revista de Enfermería. Universidad Albacete, Septiembre; 6: 6 -19

- Comunidad de Madrid (1997) Hábitos de salud en la población juvenil de la Comunidad de Madrid. Boletín epidemiológico de la Comunidad de Madrid. 5(8): 3-17.

- GARCÍA J. PEREIRO I. PÉREZ E. (1997) Diferencias existentes entre las adolescentes atendidas en primera visita en un centro de planificación familiar en dos periodos de tiempo. Atención Primaria. Vol. 20, n'5 septiembre; 237-241.

- IBARS PRUNÉS, J. OLLÉ RAMOS, N. PEIRÓ FORNAS, N. PERALTA PUIG, MC. (1998) Adolescentes y centros de planificación familiar. Enfermería clínica, 1; 8 .

- JIMÉNEZ LLORENTE Y COLS. (2000) Embarazo en adolescentes y conocimientos sobre sexualidad en la Zona Básica Bajo Andarax. Centro de Salud, marzo; 164-169.

- JIMENO, C. (2001) Llega el "día después". Salud entre todos (Consejería Andaluza de Salud). Invierno; 86: 14-19.
- LERTXUNDI R. La Adolescencia en el 2000. Clínica Euskaldura Bilbao.

- MUÑOZ GALLiGO, E. MATTOS GUILLÉN, I. PERAMO MOYA, B. (1999) Anticoncepción y sexualidad en la adolescencia. Toco-ginecología práctica, 635; 58: 81-87.

- OLTRA RODRÍGUEZ E., GONZÁLEZ ALLER C., CORTÉS PINTO R. (1994) "Trabajo con jóvenes en atención primaria de salud" $1^{\text {a }}$ Reunión asturiana (monografía).Octubre 94.

- PELÁEZ MENDOZA, J. RODRÍGUEZ PONS, O. BERMÚDEZ SÁNCHEZ, R. (2000) Adolescente varón y anticoncepción. Toco-ginecología práctica, 645; 59: 189-193.

- RODRÍGUEZ DE LA PARRA, S. CARBELO BAQUERO, B. (1999) Autocuidado en el adolescente. Revista Rol de enfermería. 1999; 22: 7-8.

- SÁNCHEZ A. (1999) "Sexo, adolescentes y cifras", una responsabilidad en salud pública. Jano, 29 de octubre - 4 de noviembre. Vol. LVII n ${ }^{\circ} 1317$.

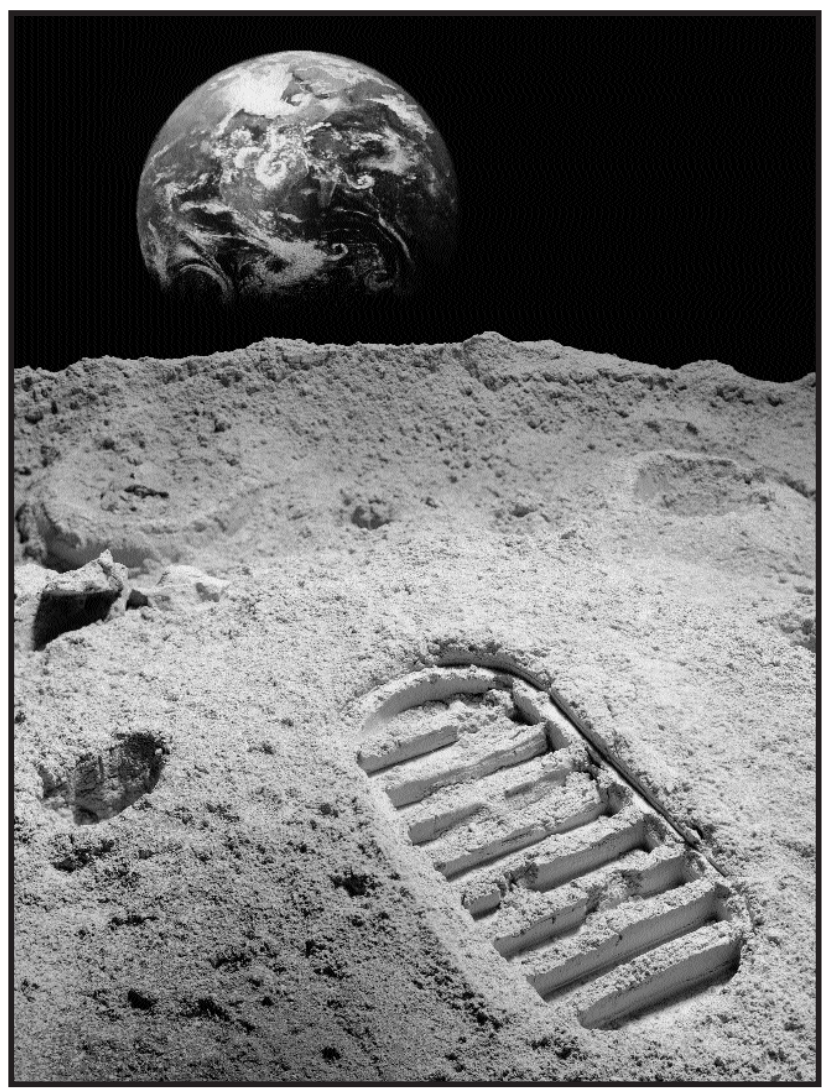

